\title{
Editorial
}

\section{Ernährung des älteren Menschen: fachübergreifend handeln, Mangelernährung vermeiden}

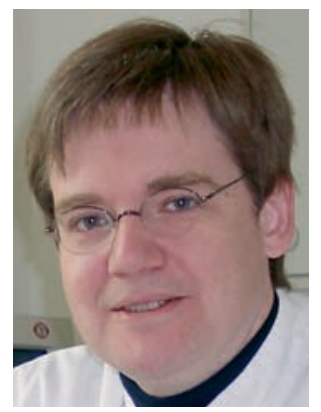

Obwohl schon seit einiger Zeit die adäquate Ernährung älterer Menschen zunehmend in den Fokus rückt, findet sich immer noch ein hoher Anteil mangelernährter Senioren, insbesondere in medizinischen Einrichtungen. Aus diesem Grund werden im Heimbereich inzwischen häufiger u.a. standardisierte Kontrollen des Ernährungsstatus älterer Patienten vorgenommen. Trotzdem stellt Mangelernährung im Alter weiterhin eine große Herausforderung dar. Die Feststellung von Mangel alleine reicht eben nicht aus, diesen auch zu beseitigen. Gefordert ist eine frühzeitige und umfassende Suche nach der Ursache. Ursachen und auch Folgen von Malnutrition sind vielfältig und manchmal nur schwer zu erkennen.

Stürze und zunehmende Mobilitätseinschränkungen sollten neben organischen Ursachen wie Arthrose oder Paresen auch an mögliche Mangelzustände mit hieraus resultierendem langsam voranschreitendem Muskelabbau denken lassen. Gerade im Alter wird auf die Eiweißreserve Muskulatur zurückgegriffen, wenn die orale Zufuhr nicht mehr ausreicht (S. 8).

Voraussetzung für eine ausreichende orale Nahrungsaufnahme ist eine intakte Mundgesundheit. Leider stehen vielfach immer noch die Inspektion des Mundraumes und die Begutachtung des Schluckaktes nicht im Vordergrund der ärztlichen und pflegerischen Befunderhebung. Jedoch sind neben offensichtlichem Zahnverlust auch Veränderungen am Kiefer oder den Mundschleimhäuten häufig Ursache der reduzierten Kalorienaufnahme.
Daneben wird weiterhin das Nebenwirkungsprofil vieler Medikamente unterschätzt, die neben der Verschlechterung des Appetits zusätzlich die sowieso schon im Alter zunehmende Mundtrockenheit verstärken und somit Erkrankungen im Mundbereich Vorschub leisten (S. 12).

Unterschätzt wird auch die Rolle des Flüssigkeitshaushaltes bei Älteren. Durch Mangel an Flüssigkeit ist die Sterblichkeit älterer Patienten deutlich erhöht und manche delirante Episode könnte durch eine rechtzeitige Intervention vermieden werden (S. 18). Um spezifische Anforderungen im Alltag geht es bei der ausreichenden Versorgung von Menschen mit demenziellen Erkrankungen (S. 28).

Mangelernährung im Alter zeigt die unterschiedlichsten Facetten aus den verschiedensten Ursachen mit mannigfaltigen Auswirkungen und häufig fatalen Folgen. Mangelernährung im Alter werden wir nur begegnen können durch eine teamorientierte, fachübergreifende Betrachtung des Problems im intensiven Dialog.

Es bleibt die Herausforderung, in interdisziplinärer und multiprofessioneller Zusammenarbeit frühzeitig Mangelernährung zu erkennen und diese gemeinsam effektiv zu behandeln. Denken wir daran!

\author{
Dr. med. Wolfrid Schröer \\ Chefarzt Geriatrie \\ Klinikum Duisburg
}

